\title{
EXTENSÃO UNIVERSITÁRIA: "USO DE EPI'S, CONSCIENTIZAÇÃO DO TRABALHADOR DA CONSTRUÇÃO CIVIL"
}

DOI: 10.37702/2175-957X.COBENGE.2021.3457

Adriana Xavier Américo - dridrikamerico@gmail.com

Universidade Federal do Pará

Tv. Éneas Martins 39

68400-000 - Cametá - PA

Julia Carolina Cavalcante de Sousa - juliaccs.1999@gmail.com

UFPA

$\mathrm{Km} 42 \mathrm{Br} 31600$

68644-000 - Santa Luzia do Pará - PA

Samilly Marques dos Praseres - millymarques29@gmail.com

Universidade Federal do Pará

Passagem São Jorge 44

68458-285 - Tucuruí - PA

EDIL DA GAMA DOS SANTOS - santosedilsantos1@gmail.com

UNIVERSIDADE FEDERAL DO PRÁ

Rua Max parijos 1400

64800-000 - Cametá - PA

Carolina Coelho da Rosa - carolinacrosa@gmail.com

Universidade Federal do Pará

Rua Juruá 17

68455-745 - Tucuruí - PA

Resumo: A extensão universitária permite que os discentes participantes criem conexões com a comunidade, e que ainda aprimorem seus conhecimentos. Neste sentido, o projeto de extensão "Uso de EPI's: conscientização do trabalhador da construção civil" tem a finalidade de desenvolver cursos e palestras para os trabalhadores da construção civil e para os estudantes de cursos técnicos e universitários. Neste trabalho foi analisada a importância das atividades do projeto para os voluntários participantes e as principais dificuldades enfrentadas na execução. Concluiu-se que o projeto permitiu que os estudantes aprofundassem o 


\section{COBENGE e IV Simpósio Internacional

conhecimento sobre a segurança do trabalho, além disso, notou-se que para os voluntários, conciliar as atividades do projeto com as demais atividades ainda é um desafio, e que, embora satisfeitos, ainda precisam melhorar a sua atuação.

Palavras-chave: voluntários, percepção, proteção, segurança do trabalho 


\section{INTRODUÇÃO}

A indústria da construção civil é de suma importância para o desenvolvimento econômico nacional, no qual contribui significativamente para o PIB e proporciona a geração de inúmeros empregos. No entanto, este setor está entre os mais susceptíveis aos acidentes de trabalho, ocupando o segundo lugar em mortes durante a execução de seus serviços, perdendo apenas para o transporte terrestre (ASSOCIAÇÃO NACIONAL DE MEDICINA DO TRABALHO, 2019).

De acordo com Amaral (2013), pesquisas demostram reduções significativas ao longo dos últimos anos no número de funcionários afastados por incapacidade laborativa e essa diminuição pode ser ainda mais considerável com a conscientização dos trabalhadores sobre a necessidade de utilizar os equipamentos de proteção individual no momento de realização das atividades laborais.

Nesta perspectiva, outro ponto importante é o conhecimento acerca dos direitos dos trabalhadores, isto é, o direito de o colaborador receber do empregador gratuitamente e em perfeito estado os EPI's necessários para desenvolver suas atividades. Mas, embora algumas empresas atendam a essas exigências, não são todos os funcionários que utilizam, portanto, o empregador deve exigir, orientar e treinar a sua equipe quanto a importância do uso adequado de tais equipamentos de proteção, visando o bem-estar de todos (WRUBEL, 2013).

Diante do exposto, foi desenvolvido o projeto de extensão "Uso de EPI's: conscientização do trabalhador da construção civil", por discentes do curso de engenharia civil da UFPA - Campus Universitário de Tucuruí (CAMTUC), com o intuito de desenvolver palestras, materiais teóricos e ilustrativos e vídeos aulas, a fim de gerar conteúdo e conscientizar a população sobre essa temática. No entanto, os benefícios do projeto vão além de seu escopo proposto inicialmente, uma vez que influência notoriamente no desenvolvimento dos voluntários envolvidos.

Dessa forma, o presente trabalho tem como objetivo avaliar a relevância e a contribuição do projeto de extensão aos voluntários deste. Sendo assim, foi analisada a percepção dos integrantes quanto ao conhecimento sobre a relevância do tema, a contribuição do projeto para cada um e as principais dificuldades encontradas durante a execução.

\section{REFERENCIAL TEÓRICO}

Os maiores desafios identificados para a saúde dos trabalhadores, em qualquer época, é o acidente do trabalho. Diante disso, é importante ressaltar que essas ocorrências levam em consideração não somente a lesão física, como também a perda de tempo e os danos materiais, ou mesmo as três coisas simultaneamente (CISZ, 2015). 
Devido à necessidade de regularização entre empregador e empregados, foram elaboradas as Normas Regulamentadoras (NR's), que em 1978 instituiu a Lei 6.514, relativas à segurança e medicina do trabalho. Dessa forma, é obrigatória sua obediência pelas empresas públicas e privadas, pelos órgãos públicos da administração direta e indireta e pelos órgãos dos Poderes Legislativo e Judiciário, que possuam empregados regidos pela Consolidação das Leis do Trabalho (CLT). Nesse sentido, é de suma importância destacar que a segurança do trabalhador não é apenas uma obrigação de cumprimento da lei, e sim uma forma de promoção do desenvolvimento e da valorização do ser humano, onde há respeito a saúde, a integridade física e o bem-estar do colaborador (SILVA et al., 2015).

Diante desse cenário, como uma forma de proteger os trabalhadores a exposição dos perigos existentes em determinadas atividades laborais, surgem os EPI's. Esses equipamentos são designados pela Norma Regulamentadora $n^{\circ} 6$ (NR-6) como "todo dispositivo ou produto, de uso individual utilizado pelo trabalhador, destinado à proteção de riscos suscetíveis de ameaçar a segurança e a saúde no trabalho" (BRASIL, 1978).

Os tipos de EPI's são diversos, com a finalidade de proteger às várias partes do corpo humano, cuja característica define a sua utilização em ambientes de acordo com a necessidade. Conforme Brandão e Rodrigues (2017), alguns dos equipamentos utilizados na construção civil são destinados à proteção da cabeça contra impactos, choques elétricos e no combate a incêndios - capacete; proteção dos olhos contra luminosidade intensa e contra impactos de partículas volantes - óculos; proteção auditiva para trabalhadores que atuam em locais com grandes níveis de ruídos - tipo concha ou tipo inserção; proteção dos membros superiores e inferiores - luvas e calçados de proteção; e outros.

Apesar dos inúmeros benefícios que os EPI's proporcionam, ainda há um entrave quanto à utilização pelos trabalhadores. Assim, esses problemas propiciam diversos acidentes nos canteiros de obras, o que, consequentemente, provoca um baixo desenvolvimento da Construção Civil. Essa ausência de uso dos EPI's pode ser explicada pela não disponibilização dos dispositivos necessários para a proteção por parte dos empregadores, ou pela resistência dos colaboradores quanto ao uso (CISZ, 2015). Portanto, é imprescindível que haja o fornecimento dos equipamentos pela empresa e a conscientização dos empregados quanto a sua importância, para que assim haja o desenvolvimento seguro e produtivo da obra.

De acordo com Costa et al. (2019), a recusa do empregado em utilizar os equipamentos de proteção se dá por diversos fatores, no qual pode-se destacar que $86 \%$ dos colaboradores utilizam EPI para cumprir as exigências da empresa, sendo notadamente visto que apenas $14 \%$ realmente acreditam na eficiência e segurança que é proporcionada por esses dispositivos de proteção. Assim como, os autores também comentam que $50 \%$ dos entrevistados relatam não ter treinamento para manipular adequadamente os EPI's.

Dessa forma, fica nítida a necessidade de treinamento e conscientização dos trabalhadores da construção civil, o que simultaneamente mostra que é possível associar os discentes do curso de engenharia civil com a melhora do desenvolvimento de uma cultura que preze a valorização da vida humana. Isso se deve à construção do aprendizado dos estudantes sobre os cuidados na realização das atividades laborais, durante a graduação. Em virtude disso, o conhecimento pode influenciar não somente na percepção da importância da segurança no trabalho, como também na disseminação de sua relevância e uso correto de equipamentos de prevenção, enraizando uma cultura prevencionista para ambos os lados. 


\section{METODOLOGIA}

O projeto de extensão "Uso de EPIs: conscientização do trabalhador da construção civil" tem como finalidade educar e conscientizar sobre a importância do uso dos Equipamentos de Proteção Individual, utilizando de estratégias para garantir a segurança e a saúde dos profissionais do setor da construção civil.

O público-alvo do projeto são trabalhadores e estudantes da área de construção civil, sendo que a participação deles é garantida por convite e por divulgação, feita pela equipe do projeto, principalmente nas empresas onde esses trabalhadores desenvolvem suas atividades laborais, e por meio das redes sociais.

Para o desenvolvimento do projeto foi necessária a criação de três equipes técnicas, todas formadas por voluntários. A primeira equipe ficou responsável pela produção de materiais teóricos. A segunda equipe assumiu a responsabilidade de produzir uma revista ilustrativa. Já a terceira e última equipe, ficou responsável pela mídia do projeto.

As atividades desenvolvidas e em desenvolvimento pela equipe técnica do projeto, são palestras e cursos, vale lembrar que as palestras são voltadas para estudantes e os cursos serão disponibilizados apenas para trabalhadores da construção civil. Em relação às palestras, no total serão realizadas três, mas somente uma já foi efetuada. Em se tratando de cursos, apenas um será realizado.

A primeira palestra ocorreu de forma totalmente virtual, para isso foram utilizadas as ferramentas sympla e google meet. A sympla é uma plataforma online onde é possível organizar eventos, por meio dela é viável que os interessados nos eventos realizem suas as inscrições, recebam o link do evento e os certificados (TORNASEL E CARTACHO, 2021). Já o google meet, é um serviço de comunicação por vídeo, onde a sociedade pode compartilhar conhecimento (SILVA et al, 2020).

Em relação ao curso, ele ainda será realizado virtualmente. Em outras palavras, a equipe técnica responsável pela produção dos materiais teóricos, irá produzir aulas divididas nos seguintes tópicos: introdução, segurança do trabalho e comissão interna, principais benefícios para empregadores e colaboradores, normas regulamentadoras, conservação e cuidados com os EPl's, por fim, conclusão e agradecimentos.

Além disso, será utilizado o software da Microsoft (Powerpoint), já que por meio dele é possível criar slides e gravar apresentações. Sendo assim, depois de gravadas as aulas serão postadas no canal do Youtube do projeto, e por fim, serão disponibilizadas em um grupo de WhatsApp, onde será enviado o link. Será disponibilizado também o material de apoio: dois Ebook e uma revista ilustrativa.

Outro ponto importante, é a forma de avaliar a atuação do projeto, dessa maneira, foi realizada uma pesquisa descritiva com os voluntários (Figura 1), para que eles pudessem expor sobre a importância do projeto e as dificuldades encontradas na execução. Cabe destacar, que as pesquisas descritivas (quantitativas e qualitativas), ocorrem por meio da aplicação de questionários (CRESWELL e CRESWELL, 2020). 
Figura 1: Questionário aplicado aos voluntários.

Em relação ao seu conhecimento sobre a importância da segurança do trabalho para a construção civil:

Já conhecia o assunto.

Conhecia, mas o projeto possibilitou ter mais conhecimento sobre o assunto e sua importância.

Conhecia por meio do projeto.

Considerando o desenvolvimento do projeto até o presente momento e sua atuação no mesmo, você se considera:

Muito satisfeito.

Satisfeito, mas sinto que devo melhorar meu envolvimento nas atividades.

Satisfeito, mas sinto que o desenvolvimento do projeto deve ser melhorado.

Não satisfeito.

Analisando o seu desenvolvimento acadêmico e profissional, você nota alguma contribuição do projeto?

Sua resposta

Agora, selecione as principais dificuldades que você enfrenta no desenvolvimento das atividades do projeto:

Não enfrento dificuldades.

Trabalho em grupo.

Conciliar o projeto com minhas demais atividades

outros

Fonte: autores (2021). 


\section{RESULTADOS E DISCUSSÕES}

O questionário foi disponibilizado por meio eletrônico e respondido por 11 voluntários do projeto de extensão, sendo todos discentes da Universidade Federal do Pará - Campus de Tucuruí e cursando o sexto semestre do curso de Engenharia Civil.

Os dados obtidos evidenciam que $91 \%$ dos integrantes entrevistados já conheciam a importância da segurança do trabalho para a construção civil, uma vez que a disciplina de Segurança na Construção Civil é ofertada no $5^{\circ}$ semestre desse curso, mas que o projeto possibilitou o ganho de mais conhecimento acerca do assunto (Gráfico 1).

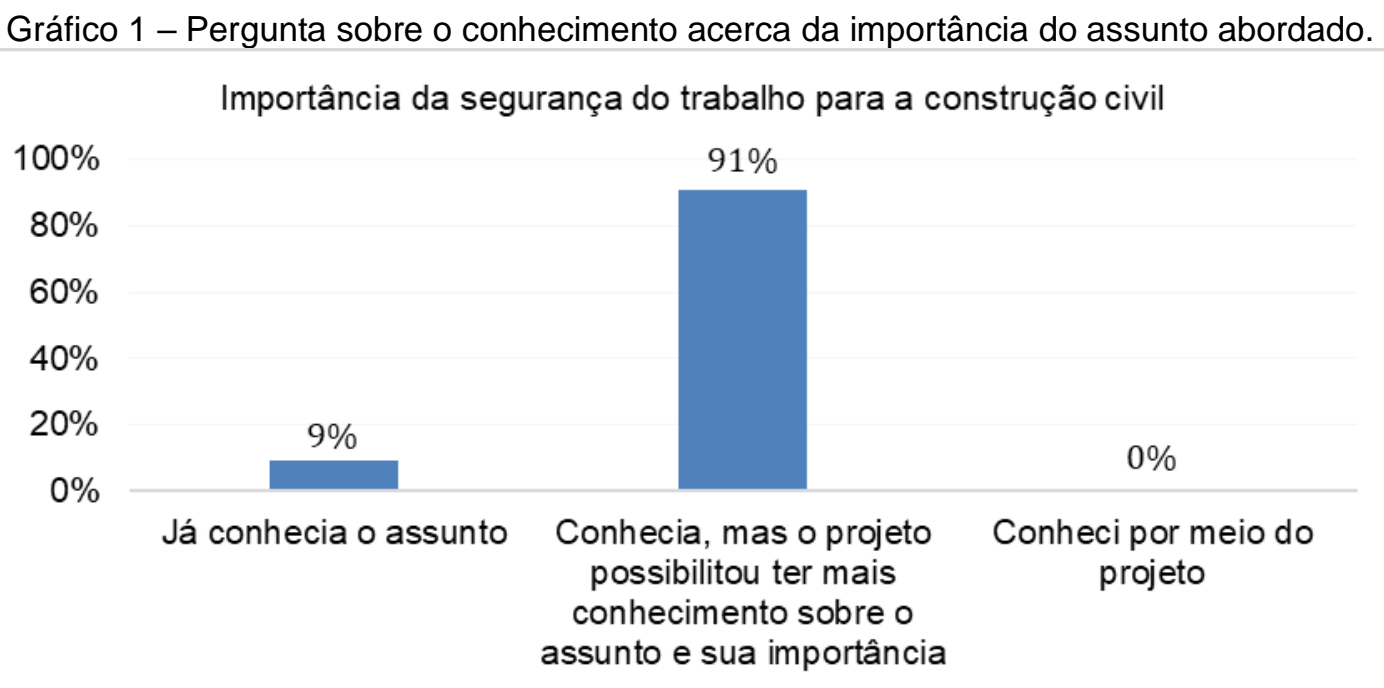

Fonte: Autores (2021).

Segundo Bridi et al. (2013) é importante que todos os profissionais do ramo da construção conheçam a importância da realização de suas atividades laborais com segurança, pois a falta de conhecimento sobre o assunto dificulta a adoção de medidas adequadas para solucionar problemas causadores de acidentes. Dessa forma, percebe-se a contribuição do projeto na formação de engenheiros comprometidos com a saúde e o bem-estar dos funcionários envolvidos nas atividades construtivas.

No que tange à contribuição do projeto para o desenvolvimento acadêmico e profissional dos membros do projeto, $81,8 \%$ dos membros notaram melhora na sua capacidade de desenvolver trabalhos em equipe, sendo citado por um dos voluntários que "surgiu a necessidade de aprender a se relacionar melhor com todo o grupo de modo a contribuir para o crescimento do projeto". Nessa perspectiva, Franco e Santos (2010) ressaltam a importância do trabalho em equipe para o desenvolvimento de qualquer atividade, pois, em suas palavras, "a interação entre as pessoas envolvidas pode se transformar na base de sustentação da organização".

Por outro lado, com relação ao desenvolvimento do projeto até o presente momento e a participação dos voluntários, $45,5 \%$ responderam que estão muito satisfeitos com ambos, 36,4\% consideram-se satisfeitos, mas acreditam que devem melhorar sua atuação na realização das atividades e 18,2\% responderam que o desenvolvimento do projeto deve ser melhorado (Gráfico 2). 
Gráfico 2 - Pergunta sobre o desenvolvimento do projeto até o presente momento.

\begin{tabular}{|c|c|c|c|c|}
\hline \multicolumn{5}{|c|}{ Desenvolvimento do Projeto } \\
\hline & & 3610 & & \\
\hline & & & & \\
\hline \multirow{3}{*}{$\begin{array}{l}30 \% \\
20 \% \\
10 \%\end{array}$} & & & 1020 & \\
\hline & & & $18,2 \%$ & \\
\hline & & & & \\
\hline \multirow{2}{*}{$0 \%$} & & & & $0,0 \%$ \\
\hline & Muito satisfeito & $\begin{array}{c}\text { Satisfeito, mas devo } \\
\text { melhorar meu } \\
\text { envolvimento nas } \\
\text { atividades }\end{array}$ & $\begin{array}{l}\text { Satisfeito, mas o } \\
\text { desenvolvimento do } \\
\text { projeto deve ser } \\
\text { melhorado }\end{array}$ & Não satisfeito \\
\hline
\end{tabular}

Fonte: Autores (2021).

A última análise foi quanto às principais dificuldades enfrentadas pelos integrantes visando o desenvolvimento de suas tarefas no trabalho de extensão. Sendo assim, 72,7\% apresentam problemas em conciliar as atividades do projeto com seus demais afazeres, $18,2 \%$ não apresentam dificuldades e 9,1\% apontaram o trabalho em grupo como fator problemático para a sua participação (Gráfico 3).

Gráfico 3 - Pergunta sobre as principais dificuldades enfrentadas no desenvolvimento do projeto.

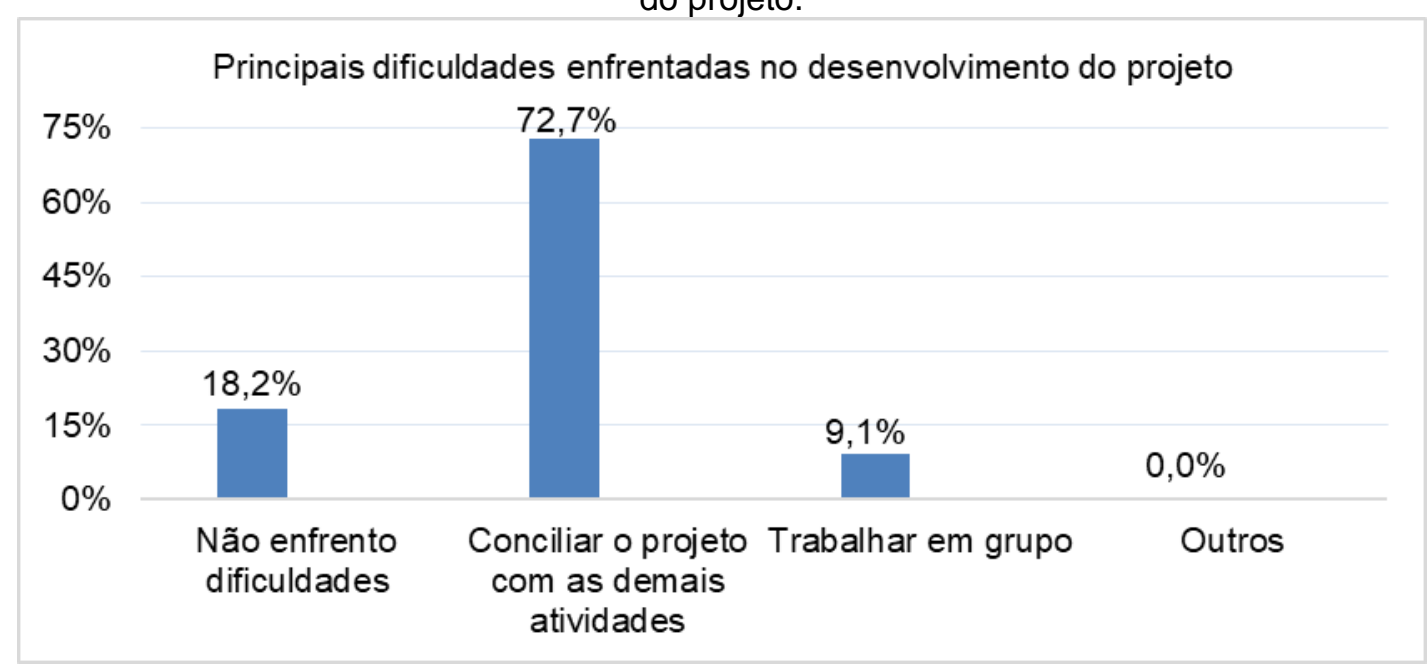

Fonte: Autores (2021).

De acordo com Oliveira et al. (2016), a capacidade de organização de tarefas e tempo é uma relevante necessidade da atualidade, no entanto, ainda é uma dificuldade apresentada por muitas pessoas, o que pode dificultar a realização exitosa de algumas ações. Dessa maneira, percebe-se a importância de que cada membro do projeto busque formas de melhorar sua organização, para que não prejudique o desenvolvimento de suas demais tarefas e para melhorar a sua atuação no projeto de extensão, gerando assim maior satisfação aos voluntários. 


\title{
5 CONSIDERAÇõES FINAIS
}

Os acidentes de trabalho na indústria da construção civil são considerados significativos, no qual exibe a necessidade de movimentos ou organizações maiores para que haja transformações quanto ao nível de importância atribuído aos dispositivos utilizados para proteção dos profissionais que atuam nessa área. Dessa maneira, os estudos e as atividades realizadas no projeto buscam amenizar esses acontecimentos tão recorrentes no ambiente laborativo, por meio da conscientização através de palestras ou cursos.

Diante disso, foi possível inferir que o projeto de extensão possibilitou ampliar a margem de conhecimentos dos voluntários acerca do assunto sobre segurança do trabalho na construção civil. Assim, sob a visão dos participantes foi demonstrado que o tema abordado no projeto é de extrema relevância para a sociedade, haja vista que, é possível difundir melhor os aprendizados sobre a segurança e a saúde dos profissionais do setor em questão. Nesse contexto, o objetivo de educar e conscientizar os colaboradores dessa indústria sobre os significativos resultados de uso de EPI's, mostrou-se viável com a metodologia proposta.

Em uma outra análise, o projeto permitiu não somente a evolução na percepção da importância do uso de EPI's pelos voluntários, como também no desenvolvimento da habilidade de trabalhar em equipe. Sendo assim, a capacidade de lidar com ideias e experiências diferentes ao longo da realização das atividades é aumentada, promovendo a união e a organização dos integrantes.

\section{REFERÊNCIAS}

AMARAL, A. G. do. Segurança no trabalho: EPI'S na construção civil. Revista Ciência Empresarial. UNIPAR, Umuarama, v. 14, n. 2, p. 231-257, jul./dez. 2013.

\begin{abstract}
ASSOCIAÇÃO NACIONAL DE MEDICINA DO TRABALHO - ANAMT. Construção civil está entre os setores com maior risco de acidentes de trabalho. [S. I.]: Associação Nacional de Medicina do Trabalho, 30 abr. 2019. Disponível em: https://www.anamt.org.br/portal/2019/04/30/construcao-civil-esta-entre-os-setores-commaior-risco-de-acidentes-de-trabalho/. Acesso em: 12 abr. 2021.
\end{abstract}

\section{BRANDÃO, I. M. T.; RODRIGUES, M. M. N.. A ineficiência na utilização dos epi's na} construção civil. 2017. 38 p. Trabalho de Conclusão de Curso (Bacharelado em Engenharia Civil) - Centro Universitário Cesmac, Maceió, 2017.

Disponível em:

https://ri.cesmac.edu.br/bitstream/tede/351/1/A\%20INEFICI\%c3\%8aNCIA\%20NA\%20UTI LIZA\%c3\%87\%c3\%830\%20DOS\%20EPI\%e2\%80\%99S\%20NA\%20CONSTRU\%c3\%87 \%c3\%830\%20CIVIL.pdf. Acesso em: 10 abr. 2021.

BRASIL. Ministério do Trabalho e Emprego - MTE. Norma Regulamentador - NR 6: Equipamento de Proteção Individual (EPI). Portaria GM oㅜ 3214, de 8 de junho de 1978 e alterações até 2018. Secretaria de Segurança e Saúde no Trabalho -SSST, 1978. 
BRIDI, M. E. et al. Identificação de práticas de gestão da segurança e saúde no trabalho em obras de construção civil. Ambiente Construído, Porto Alegre, v. 13, n. 3, p. 43-58, 2013.

CISZ, C. R. Conscientização do uso de EPI's, quanto à segurança pessoal e coletiva. 2015. 44 p. Dissertação (Pós Graduação em Engenharia de Segurança do Trabalho) Universidade Tecnológica Federal do Paraná, Curitiba, 2015. Disponível em: http://repositorio.utfpr.edu.br/jspui/bitstream/1/17681/2/CT CEEST XXIX 2015 07.pdf.

Acesso em: 10 abr. 2021.

COSTA, D. M.; DIAS, V. de S.; ARÃO, I. R. Motivos que levam o trabalhador da construção civil a deixar de usar os equipamentos de proteção individual. Revista Interdisciplinar de Pós-graduação da UniAraguaia, v. 2, n. 1, p. 78-87, 2019.

CRESWELL, J. W.; CRESWELL, J. D. Projeto de pesquisa: Métodos qualitativo, quantitativo e misto. Penso Editora, 2021.

FRANCO, J. H. S.; SANTOS, J. N. Um estudo da relação entre o trabalho em equipe e a aprendizagem organizacional. Revista Eletrônica Gestão e Sociedade, Belo Horizonte, v. 4, n. 10, p. 736-756, 2010.

OLIVEIRA, C. T. et al. Oficinas de gestão do tempo com estudantes universitários. Psicologia: Ciência e Profissão, Brasília, v. 36, n. 1, p. 224-233, 2016.

SILVA, A. P. D. et al. A importância da Gestão da Segurança na Construção Civil com vistas à saúde, medicina do trabalho e Conscientização dos Trabalhadores: Um Estudo de caso na Construtora cv lopes Itda. Fórum de Administração, v. 6, n. 1, 2015.

SILVA, B. A. D; FRANCO, A. P. C. L; COELHO, S. F; CASTRO, M. Ensino remoto: análise comparativa do zoom e do google meet no contexto educacional. Congresso internacional de linguagem e tecnologia online, 2020. Anais. Disponível em: http://www.periodicos.letras.ufmg.br/index.php/anais linguagem tecnologia/article/view/17 836. Acesso em: 17 abr. 2021.

TORNASEL, D; CARTACHO, M. Softwares. Disponível em: https://www.sympla.com.br/. Acesso em: 17 abr. 2021.

WRUBEL, A. A utilização de EPI'S na construção civil: uma abordagem em duas construtoras de Curitiba. Trabalho de conclusão de curso - Curso de Tecnologia em Concreto. Universidade Tecnológica Federal do Paraná Campus Curitiba, Curitiba, 2013.Disponível em: http://repositorio.roca.utfpr.edu.br/jspui/bitstream/1/2072/1/CT TCC 20131 02.pdf. Acesso em: 11de abril de 2021. 


\title{
UNIVERSITY EXTENSION: “USE OF PPE: AWARENESS OF CIVIL CONSTRUCTION EMPLOYEES"
}

\begin{abstract}
The university extension allows participating students to create connections with the community, and to further enhance their knowledge. In this sense, the university extension project "Use of PPE: awareness of civil construction employees" aims to develop courses and lectures for construction workers and students of technical and university courses. In this work, the importance of the project activities for the participating volunteers and the main difficulties faced in the execution were analyzed. It was concluded that the project allowed students to deepen their knowledge about work safety, in addition, it was noted that for volunteers, reconciling project activities with other activities is still a challenge, and that, although satisfied, still need to improve their performance.
\end{abstract}

Keywords: volunteers, perception, protection, work safety. 